\title{
Fat embolism syndrome - a case report with literature review
}

\begin{abstract}
Background: The fat embolism syndrome (FES) has been considered a diagnostic enigma since its first description over a century ago. Varying degree of presentation along with absence of specific diagnostic criteria or a scoring system makes it difficult to be diagnosed. Simple fat embolism may be a pathologic finding with variable clinical significance; patients with the FES have fat emboli in multiple organs, causing extensive organ dysfunction. While most patients with FES fully recover, there is an estimated 5to15percent mortality rate. There is no wide accepted specific treatment beyond aggressive supportive care.
\end{abstract}

Case report: A 72year old Caucasian female presented with dyspnea, hypoxia and altered mental status within 24 hours of admission for an inferior pubic rami fracture secondary to fall. Patient required intubation and mechanical ventilation for respiratory failure. An Echocardiogram revealed Right Ventricular strain and MRI of the brain showed multiple acute/subacute infarcts, which supported a diagnosis of FES, especially as pulmonary embolism was deemed less likely with a negative CT Chest Angiogram and US Doppler of Legs. The patient gradually recovered with supportive care, successfully extubated with eventually discharged after thirty days of hospitalization.

Conclusion: Fat emboli syndrome has a variable degree of presentation, ranging from mild symptoms to severe multi $\neg$ organ involvement that can ultimately be fatal. It can also be associated with long $\neg$ term morbidity which emphasizes the need for rapid precise diagnosis and management.

Keywords: fat embolism syndrome, pelvic ramus fracture, multi $\neg$ organ, echocardiogram, caucasian female, morbidity
Volume 3 Issue 3 - 2015

Johnson Gomez, IshaVerma, Maria Angela
Landicho, Abhinav Agrawal,Violet Kramer
Department of Medicine, Monmouth Medical Center, USA

Correspondence: Abhinav Agrawal, Department of Medicine, Monmouth Medical Center, 300 Second Avenue, Long Branch, NJ 07740, USA, Email abhinav72@gmail.com

Received: October 27, 2015 | Published: December 04, 2015
Abbreviations: FES, fat embolism syndrome; PFE, pulmonary fat embolism; PFO, patent for men ovale; FFA, free fatty acids; CRP, $\mathrm{C}$ reactive protein; IL6, interleukin6

\section{Introduction}

The fat embolism syndrome (FES) has been considered a diagnostic enigma since it was first described a century ago. It has continued to beach allenging diagnosis to secured espite many technologic advances. The difficulty with which to diagnose FES stems from the fact that it can complicate an array of clinical presentation with a variable severity of illness. Although simple fat embolism may be a pathologic finding with little clinical significance, patients with the fat emboli syndrome have fat emboli in multiple organs, which show extensive damage from this embolization. ${ }^{1}$ While most patients with FES fully recover, there is still an estimated5to 15 mortality risk. ${ }^{2}$ Care is generally supportive. We report a case of a Caucasian female with a near fatal fat embolism syndrome with multiorgan involvement in the setting of a nondisplaced traumatic pelvic ramus fracture.

\section{Case background}

\section{Patient information}

A 72yearold Caucasian female with a history of Type two diabetes mellitus, hypertension, hyperlipidemia, anxiety, gastro paresis and chronic back pain presented with acute respiratory distress, hypotension and altered mental status around twelve hours after sustaining fall which caused a traumatic pelvic ramus fracture. Her family history is notable for renal failure in her mother. Her social history is significant for remote history of smoking 30years ago. There was no history of alcohol or recreational drug abuse. She lived alone and was independent with activities of daily living. She had been a garment distributor and had sold computers but was currently on disability for at least seven years.

\section{Objectives for case reporting}

FES may complicate an array of clinical entities and presents a diagnostic challenge with significant risk for morbidity and mortality. Although most case reports have described full recovery, there is definite potential for long term morbidity associated with nearlethal cases of FES. This emphasizes the importance for high clinical suspicion and rapid diagnosis for developing a more rapid and precise diagnostic and therapeutic management.

Main medical problem: Fat embolism syndrome

\section{Coexisting diseases:}

i. Diabetes mellitus type 2

ii. Hypertension Hyperlipidemia Gastroparesis Anxiety

iii. Chronic back pain

\section{Case presentation}

Our patient presented with left sided rib pain secondary to a 
mechanical fall. She also complained of pain on movement of both lower extremities. On initial presentation she was a wake, alert and oriented with stable vital signs. Initial examination revealed left leg superficial bruises and a decreased range of motion of both lower extremities. The rest of the physical examination was unremarkable. Radiographic imaging revealed left seventh rib fracture and a minimally displaced fracture of the left inferior pubicramus (Figure 1). Shortly after admission, she acutely developed lethargy, altered mental status, hypotension with blood pressure of $80 / 50 \mathrm{mmHg}$ and tachycardia with heart rate of 110 beats per minute. Pulseoximetry showed $82 \%$ oxygen saturation on four liters of oxygen via nasal cannula. The patient was transferred to the intensive care unit for close monitoring. There was transient clinical improvement with administration of bi-level positive airway pressure and fluid resuscitation.

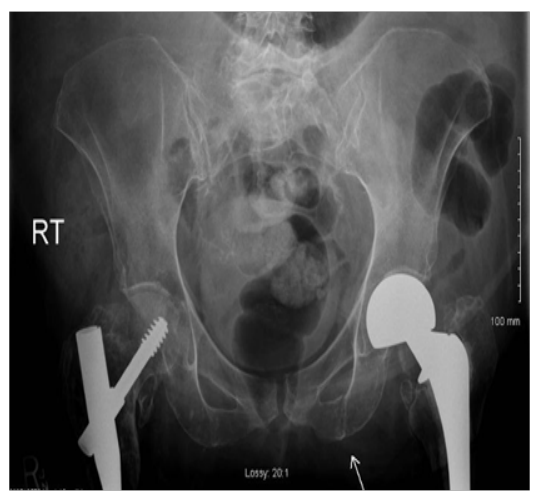

Figure I Left inferior pubic ramus fracture, nondisplaced, from a mechanical fall.

Subsequently, she had worsening dyspnea and hypoxia and was intubated. She remained hypotensive despite fluid boluses. Therefore, she was started on vasopressors. Initial laboratory findings included a normal leukocyte count and an elevated CRP of 67.4. Portable chest $\mathrm{x}$-ray showed stable pulmonary vascular congestion but scattered pulmonary in filtrates. EKG showed sinus achy cardia with right fascicular block. There were mode stelevations of Troponin with a peak value of 4.7. Her initial arterial blood gas was interpreted as an acute uncompensated respiratory acidosis. An echocardiogram displayed findings of right ventricular dilatation with a pulmonary artery systolic pressure of $3040 \mathrm{~mm} \mathrm{Hg}$, Dshaped left ventricle and dilated inferior venacava, all suggestive of acute right ventricular pressure and volume over load (Figure 2). Anecho cardiogram performed 5monthsearlierrevealed normal right ventricular pressures without signs of failure. Cardiac catheterization was not performed as it was unlikely that myocardial ischemia was from anatomic obstructive coronary disease but rather was likely a result of global hypo perfusion.

Pulmonary embolism was strongly suspected, therefore a heparin infusion was initiated and a contrast enhanced chest CT scan was performed. This demonstrated bilateral pulmonary infiltrates and effusion but did not reveal any findings of pulmonary embolus. Doppler ultrasound of the lower extremities did not show evidence of thrombosis. Heparin was then discontinued. The platelet count which was initially normal, decrease dove several days to anadir of 96,000 per micro liter but then normalized. Serotonin release assay and platelet factor 4 antibody assay were negative. Urine stained with Oil Red $\mathrm{O}$ was negative for findings of fat globules. Initial head CT did not reveal any acute hemorrhages or signs of chronic infarcts. However, a repeat $\mathrm{CT}$ scan four days after admission revealed hypodensity in the posterior right temporal lobe consistent with an infarct. A subsequent MRI of the brain showed multiple acute infarcts in bilateral water shed areas, thought to be most likely due to atherothromboemboli (Figure 3). Supportive management included intravenous fluids, enteral nutrition, vasopressors and mechanical ventilation. A diagnosis off at emboli syndrome was established after careful consideration of results from multiple laboratory and imaging studies along with the patient's initial presentation and clinical course. With supportive care, she was weaned off vasopressors after 2 weeks in the intensive care unit. A follow up echocardiogram per formed after seven days showed normalization of right ventricular size and function. She demonstrated digress of residual confusion when she was transferred to a subacute rehabilitation facility after twenty three day so hospitalization. A subsequent admission several weeks later was reported after patient was still confused disoriented and perceived to be depressed and dehydrated. Condition improved after medical management but with preserved minimal residual cognitive deficit. She has not required any orthopedic procedures for the pelvic ramus fracture.
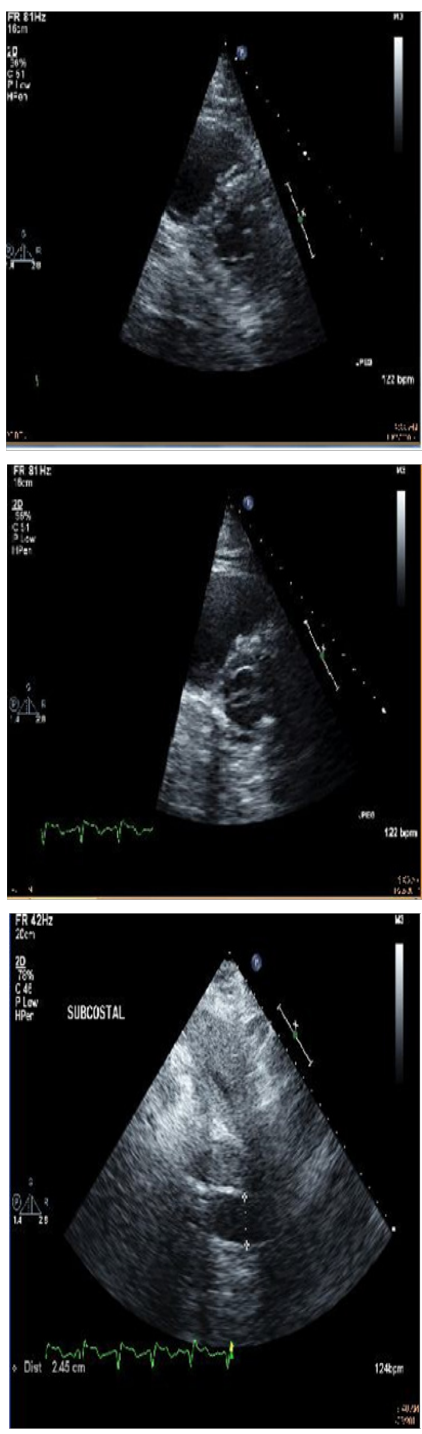

Figure 2 Transthoracic echocardiogram showing images of dilated right ventricle with straightening of interventricular septum and the Dsign (top and center) and dilated inferiorvena cava (bottom). 

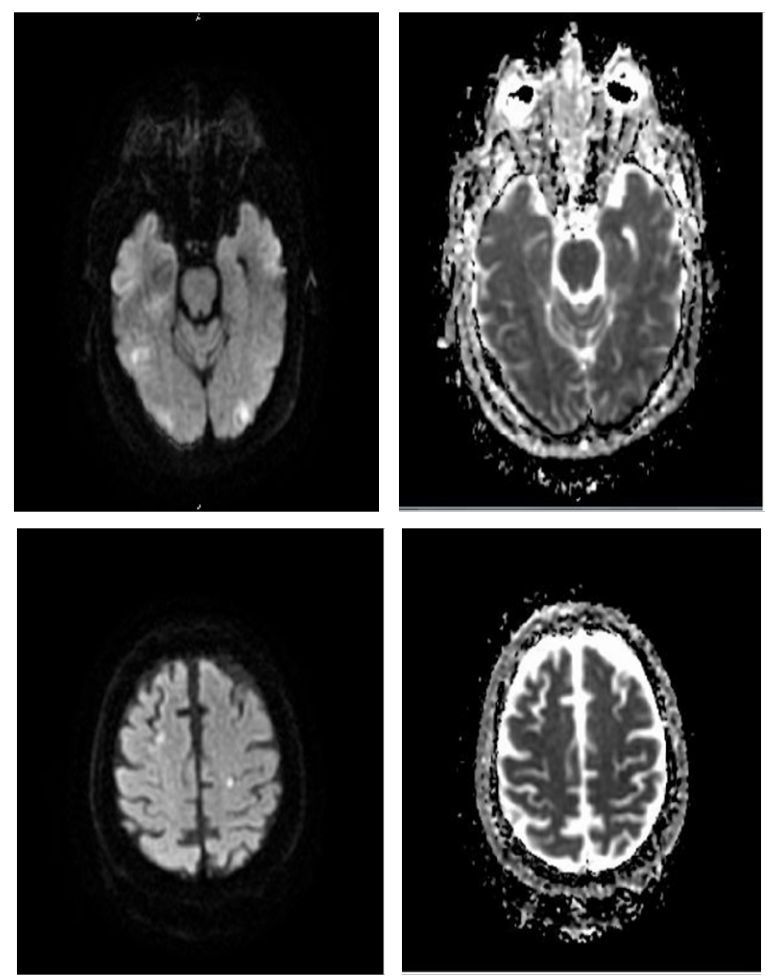

Figure $3 \mathrm{MRI}$ of the brain showing atherothrombotic emboli in bilateral watershed areas. Diffusion weighted image at level 13 (top left) with corresponding nondiffusion weighted image (top right); level 25 (bottom left) with corresponding nondiffusion image (bottom right).

\section{Discussion}

The initial observation of pulmonary fat embolism (PFE) is credited to Zenker ${ }^{3}$ in 1862. Recognition of the clinical fat embolism syndrome (FES) soon followed. ${ }^{4}$ FES is most commonly associated with long bone and pelvic fractures but it can also be associated with nontraumatic conditions such as pancreatitis, osteomyelitis, lipid infusion and bone tumourlysis. Interestingly, it is seen more frequently in closed fractures than open fractures and risk increases in proportion with the number of bone structures involved. Inone series, over 85 percent of all patients who received cardiopulmonary resuscitation had evidence of fat embolism. ${ }^{5}$

Two mechanisms regarding the pathophysiology of FES are commonly cited: mechanical and biochemical. However, these mechanisms are not necessarily mutually exclusive. ${ }^{6}$ The former presumes that fractures of marrow containing bone have the highest incidence of FES due to disrupted venules in the marrow which are tethered open by osteous attachments that allows easy entry of marrow contents into the circulation. ${ }^{7}$ From there on, it can enter the arterial circulation via a patent for men ovale (PFO) causing increases in pulmonary artery and right heart pressures or by micro embolism via the lung demonstrated even in the absence of a PFO; the latter could explain the neurologic disease and petechiae associated with FES. ${ }^{8,9}$ The bio chemical theory or mechanism explains that destabilization of a plasma chylomicronemulsion lead stocoalescence off at stores. ${ }^{10,11}$ Thereafter, degradation of embolized fat into toxic intermediaries, such as free fatty acids (FFA) and Creactive protein (CRP), results in the development of FES because FFAs are toxic to endothelial cells and pneumocytes. ${ }^{10}$ In addition, interleukin6 (IL6) may be responsible for the fever seen in FES. ${ }^{10}$ IL6may be increased due to increased production of tumor necrosis factor $\alpha$ and IL $1 \beta$, possibly related to soft tissue injury. ${ }^{10} \mathrm{CRP}$ level in FES is increased and is related to lipid agglutination (chylomicrons and LDL), which may obstruct blood flow in the microvasculature. ${ }^{12}$

FES manifests 24 to 72 hours after the initial insult, but may occur as early as 12 hours or a slate as two weeks after the inciting event. ${ }^{13}$ The classic presentation is associated with the clinical trial of dyspnea, mental confusion and petechiae. Pulmonary squealae associated with FES represent the most clinically significant change, with arterial hypoxemia being the earliest indicator of significant FES. It remains the cornerst one to the early recognition of FES. Dyspnea and tachypnea is the other common, but certainly not specific findings. ARDS is often characteristically seen,,$^{14}$ although a subacute pattern can be also observed. Approximately one-half of patients with FES caused by long bone fractures develop severe hypoxemia and require mechanical ventilation. ${ }^{15}$ Neurologic manifestation ranges from a transient and fully reversible confusion alstate and altered level of consciousness to seizures and focal deficits. ${ }^{16} \mathrm{It}$ is present in as many as 85 percent of those who succumb to FES..$^{15,16}$ The petechial rash occurs in 20 to50 percent of cases, lasts for five to seven days ${ }^{17}$ and usually develops in the head, neck, anterior thoracic, axillary and subconjunctival regions.

The diagnosis of FES rests upon the identification of symptomatology in the appropriate clinical setting in the absence of an alternative explanation. There is not a rapid and/or definitive diagnostic tool available. Chest radiographs are normal in the majority of patients ${ }^{18}$ although findings of diffuse bilateral infiltrates might paint a picture that is compatible with ARDS. Ventilation/perfusion scans may demonstrate a mottled pattern of sub segmental perfusion defects with a normal ventilation pattern. ${ }^{19} \mathrm{CT}$ of the chest may show focal areas of ground glass opacification with inter lobar septal thickening. ${ }^{20,21}$ MRI of the brain may reveal high intensity T2 signal, which correlates with the degree of clinical neurologic impairment. ${ }^{22}$ There is uncertain significance in demonstrating fat globules in urine, serum, orsputum. Study off atladen pulmonary macrophages obtained by bronchoalveolar lavage, although originally promising, ${ }^{23,24}$ has been shown to be nonspecific. . $^{25,26}$

Supportive care remains the mainstay of therapy for FES. Case reports or series have described steroids use in for life threatening cases. No specific recommendations exist regarding steroid dosing and timing. ${ }^{27,29}$ Most patients with FES fully recover, although long-term morbidity, when present, is usually in the form of cerebral neurologic deficits. ${ }^{16,30}$ The mortality rate of complicated FES is 5 to 15 percent. Our patient required an extensive ICU care and had a prolonged recovery time despite all aggressive supportive measures. This cause of prolonged recovery is likely multifactorial including the patient's comorbidities, the area of the fracture and FES itself. The decision not to administer steroid was due to lack of strong recommendations evidence that it would provide impact on the patient's outcome especially given the known risks of steroid administration. The diagnosis of FES can be concretely applied to the case due to the overall nature, symptomatology, diagnostic reports and outcome of this clinical case. The respiratory presentation that developed within 24hours of the inciting event fits the description of an acute respiratory failure and nonspecific changes in the chest radiograph and chest CT scan. Respiratory failure shortly after apelvic fracture, particularly with shock and echo cardiographic findings of RV volume and 
pressure overload make pulmonary embolism high in the differential diagnosis. This was ruled out with a negative chest CT and negative venous dopplers. Furthermore, there was no improvement with heparin therapy. Right ventricular infarction could also explain the elevated Troponin and elevated right ventricular pressure. However, the echocardiogram and EKG findings did not support the diagnosis of acute coronary syndrome and the subsequent normalization of RV function rules out a RV infarct. An infectious etiology of shock and respiratory failure was less likely given the absence of fever or leucocytosis; Furthermore, infection would not explain the elevated RV pressures. Heparin induced thrombocytopenia was considered in the light of the thrombocytopenia. However, there was no recent previous exposure to heparin and the platelet count started to decrease less than 4days from start of heparin therapy. Moreover, both serotonin release and platelet factor 4 antibody assays were negative. The neurologic symptoms, radiologic findings and sequelaeal so support the diagnosis of FES. The CT scan of the head and the MRI of the brain provided evidence of an embolic stroke involving the water shed areas. Furthermore, the imaging characteristic of the senew lesions were proposed to be athero thrombotic, which would support the diagnosis of FES. Although no petechial skin lesions or fat particles in body fluids were discovered, these findings are not essential for the diagnosis of FES, being found in only 2025 percentage of cases.

\section{Conclusion}

Fat emboli syndrome can have a highly variable presentation ranging from mild symptoms to severe multiorgan involvement that can ultimately lead to death, although the reisa relatively low reported mortalityrate.There is no specific diagnostic criteria or scoring system currentlya vailable. Furthermore, there is no reliable immediate laboratory marker or diagnostic procedure that can help confirm the diagnosis, albeitinterleukin6 assay-couple with a strong supportive management could certainly provide a promising path towards a better recognition and therapy for FES.

\section{Acknowledgements}

None.

\section{Conflict of interest}

The author declares no conflict of interest.

\section{References}

1. Dines DE, Burgher LW, Okazaki H. The clinical and pathologic correlation of fat embolism syndrome. Mayo Clin Proc. 1975;50(7):407-411.

2. Mellor A, Soni N. Fat embolism. Anesthesia. 2001;56(2):145-154.

3. Zenker FA. Beiträge zur normalen und pathologischen Anatomie der Lunge. Dresden J Braunsdorf. 1862.

4. Chan KM, Tham KT, Chiu HS, et al. Post-traumatic fat embolism-its clinical and subclinical presentations. J Trauma. 1984;24(1):45-49.

5. Eriksson EA, Pellegrini DC, Vanderkolk WE, et al. Incidence of pulmonary fat embolism at autopsy:an undiagnosed epidemic. J Trauma. 2011;71(2):312-315.

6. Dudney TM, Elliott CG. Pulmonary embolism from amniotic fluid, fat, and air. Prog Cardiovasc Dis. 1994;36(6):447474.

7. Warthin AS. Traumatic lipaemia and fatty embolism. Int Clinics 1913;23(4):171-227.

8. Pell AC, Hughes D, Keating J, et al. Brief report: fulminating fat embolism syndrome caused by paradoxical embolism through a patent foramen ovale. N Engl J Med. 1993;329(13):926-929.
9. Sulek CA, Davies LK, Enneking FK, et al. Cerebral microembolism diagnosed by transcranial Doppler during total knee arthroplasty:correlation with transesophageal echocardiography. Anesthesiology. 1999;91(3):672-676.

10. Ten Duis HJ. The fat embolism syndrome. Injury. 1997;28(2):7785.

11. Lehman EP, Moore RM. Fat embolism: including experimental production without trauma. Arch Surg. 1927;1927 14:621662.

12. Hulman G. Pathogenesis of nontraumatic fat embolism. Lancet. 1988;1:1366-1367.

13. Carr JB, Hansen ST. Fulminant fat embolism. Orthopedics. 1990;13(2):258-261.

14. Wertzberger JJ, Peltier LF. Fat embolism: the importance of arterial hypoxemia. Surgery. 1968;63:626-629.

15. King MB, Harmon KR. Unusual forms of pulmonary embolism. Clin Chest Med. 1994;15:561-580.

16. Jacobson DM, Terrence CF, Reinmuth OM. The neurologic manifestation of fat embolism. Neurology. 1986;36(6):847-851.

17. Kaplan RP, Grant JN, Kaufman AJ. Dermatologic features of fat embolism syndrome. Cutis. 1986;38(1):52-55.

18. Glas WW, Grekin TD, Musselman MM. Fat embolism. Am J Surg. 1953;85(3):363-369.

19. Park HM, Ducret RP, Brindley DC. Pulmonary imaging in fat embolism syndrome. Clin Nucl Med. 1986;11(7):521-522.

20. Arakawa H, Kurihara Y, Nakajima Y. Pulmonary fat embolism syndrome: CT findings in six patients. J Comput Assist Tomogr. 2000;24(1):24-29.

21. Malagari K, Economopoulos N, Stoupis C, et al. High-resolution CT findings in mild pulmonary fat embolism. Chest. 2003;123(4):11961201.

22. Takahashi M, Suzuki R, Osakabe Y, et al. Magnetic resonance imaging findings in cerebral fat embolism: correlation with clinical manifestations. J Trauma. 1999;46(2):324-327.

23. Benzer A, Ofner D, Tötsch M, et al. Early diagnosis of fat embolism syndrome by automated image analysis of alveolar macrophages. J Clin Monit. 1994;10(3):213-215.

24. Ch Chastre J, Fagon JY, Soler P, et al. Bronchoalveolar lavage for rapid diagnosis of the fat embolism syndrome in trauma patients. Ann Intern Med. 1990;113(8):583-588.

25. Stanley JD, Hanson RR, Hicklin GA, et al. Specificity of bronchoalveolar lavage for the diagnosis of fat embolism syndrome. Am Surg. 1994;60(7):537-541.

26. Vedrinne JM, Guillaume C, Gagnieu MC, et al. Bronchoalveolar lavage in trauma patients for diagnosis of fat embolism syndrome. Chest. 1992;102(5):1323-1327.

27. Kubota T, Ebina T, Tonosaki M, et al. Rapid improvement of respiratory symptoms associated with fat embolism by high-dose methylpredonisolone:a case report. J Anesth. 2003;17(3):186-189.

28. Fischer JE, Turner RH, Herndon JH, et al. Massive steroid therapy in severe fat embolism. Surg Gynecol Obstet. 1971;132(4):667-672.

29. Ashbaugh DG, Petty TL. The use of corticosteroids in the treatment of respiratory failure associated with massive fat embolism. Surg Gynecol Obstet. 1966;123(3):493-500.

30. Moylan JA, Birnbaum M, Katz A, et al. Fat emboli syndrome. J Trauma. $1976 ; 16(5): 341-347$. 